\title{
Markus Rieger-Ladich
}

\author{
„SCHIZOIDE DISPOSITION“ ODER „GESPALTENER \\ HABITUS"? \\ EINE PÄDAGOGISCHE LEKTÜRE VON FRANZ KAFKAS \\ BRIEF AN DEN VATER
}

Für Jörg Pramschüfer*

Franz Kafkas Brief an den Vater ist in der Vergangenheit mit den unterschiedlichsten Etiketten versehen worden: Der Erziehungswissenschaftler Klaus Mollenhauer zählte den Brief, der nie abgeschickt wurde, zu den „erstaunlichsten pädagogischen Dokumenten unserer Kultur“, der überraschende Einblicke in Erziehungsvorgänge und Bildungsprozesse gewähre ${ }^{1}$; dem Literaturwissenschaftler Christian Schärf hingegen gilt er als „einzigartiges familientherapeutisches Dokument", das aus einer sehr genauen, psychologisch geschulten Beobachtung der bürgerlichen Familie hervorgegangen sei ${ }^{2}$; der systemische Familientherapeut Helm Stierlin wiederum wendet sich dem Text zu, weil dieser nicht nur die unterschiedlichen double-bind-Situationen verzeichne, in denen sich Kafka immer wieder verstrickt habe, sondern auch den $\mathrm{Zu}$ gang zu dessen ,,innerer Landkarte““ ermögliche - zu jener Größe also, die das Wahrnehmen, Erleben und Handeln einer Person organisiert. ${ }^{3}$

So unterschiedlich diese Lektüren im Einzelnen angelegt sind, gemeinsam ist ihnen doch, dass sie den in seiner handschriftlichen Fassung mehr als einhundert Seiten umfassenden Brief eng an die Familienkonstellation rückbinden und auf diese Weise Aufschlüsse über die Beziehung zwischen Franz Kafka, dem erstgeborenen Sohn, und Hermann Kafka, dem Vater, erhoffen. Die Gründe für das offensichtlich komplizierte Verhältnis zwischen Vater und Sohn werden hier fast durchgängig innerhalb der Familie gesucht. Dabei un-

* ... in dessen Unterricht ich vor zwanzig Jahren zum ersten Mal auf Franz Kafka stieß und dem es immer wieder gelang, Begeisterung gerade auch für jene literarischen Texte $\mathrm{zu}$ wecken, die sich als etwas sperrig und spröde erwiesen.

1 Klaus Mollenhauer: Vergessene Zusammenhänge. Über Kultur und Erziehung, Weinheim, München 1998, S. 9.

2 Christian Schärf: Franz Kafka. Poetischer Text und heilige Schrift, Göttingen 2000, S. 34f.

3 Helm Stierlin: Individuation und Familie. Studien zur Theorie und therapeutischen Praxis, Frankfurt/Main 1989, S. 193. 
terscheiden sich die einzelnen Lesarten freilich erheblich hinsichtlich der Anleihen, die sie bei psychologischen oder psychoanalytischen Theoriemodellen machen: Die Bandbreite reicht hierbei von Ansätzen, die von der Adressierung individueller Verantwortung völlig absehen und statt dessen für die weitreichenden Folgen gestörter familialer Kommunikationsformen sensibilisie$\mathrm{ren}^{4}$, bis hin zu jenen Positionen, die vor einer Pathologisierung des Verfassers nicht zurückschrecken und Franz Kafka unumwunden eine „schizoide Disposition" attestieren. ${ }^{5}$

Es überrascht nun nicht, dass auch innerhalb der pädagogischen Lektüren ${ }^{6}$ jene dominieren, die einen Zugang zu dem Brief an den Vater über die Analyse der spannungsreichen Beziehung zwischen Vater und Sohn suchen. So berechtigt und hilfreich Lektüreverfahren zweifellos sind, die sich an den Persönlichkeitsmerkmalen von Hermann und Franz Kafka orientieren, die den Idiosynkrasien der beiden zentralen Akteure nachspüren und die Einzigartigkeit dieser Konfrontation zweier sehr unterschiedlicher Charaktere herausstellen - sie stehen dabei doch in der Gefahr, sich stets auf die immer gleichen Facetten zu konzentrieren und andere Einflussgrößen zu vernachlässigen. Für meine eigene Lektüre von Franz Kafkas Brief ist nun der Verdacht leitend, dass hier Auseinandersetzungen ihre Spuren hinterlassen haben, die gerade nicht in den Blick gelangen, wenn der Fokus einzig auf die Individuen Franz und Hermann Kafka gerichtet wird. Denn der Brief lässt sich eben auch interpretieren - so die These, die ich im Folgenden zu plausibilisieren versuche als ein Text, der jene Form der Entfremdung peinlich genau dokumentiert und schonungslos festhält, die sich zwischen zwei Individuen ausprägt, die in sehr unterschiedlichen Segmenten des sozialen Raumes situiert sind und in der Folge völlig unterschiedliche Habitus ausprägen. Die von Franz Kafka immer wieder beklagte Fremdheit im Verhältnis zu seinem Vater und die schmerzlich vermisste Vertrautheit scheint auf diese Weise der Dynamik des sozialen Raumes geschuldet - und eben nicht persönlichem Versagen oder individuellen Unzulänglichkeiten. Um diese verdeckten Kräfte herausarbeiten und ihre Spuren im Text dechiffrieren zu können, greife ich auf Arbeiten des französischen Kultursoziologen Pierre Bourdieu zurück. Bevor ich diesen alternativen Zugang zu Franz Kafkas Brief an den Vater jedoch erprobe (3) und den Ertrag für die erziehungswissenschaftliche Reflexion abzuschätzen versuche (4), stelle ich drei interessante und reizvolle pädagogische Lektüren des Briefes vor (1) und skizziere knapp Bourdieus Entwurf einer Theorie des sozialen Raumes (2). Ausdrücklich sei dabei eingeräumt, dass es sich bei diesem Vorgehen keineswegs um eine alternativlose Lektüre handelt: Vielmehr gilt es - wie bei der

4 Vgl. hierzu etwa: Gregory Bateson: Ökologie des Geistes. Anthropologische, psychologische, biologische und epistemologische Perspektiven, Frankfurt/Main 1992, bes.: S. 270-301.

5 Vgl.: Ch. Schärf: Franz Kafka, S. 56.

6 Vgl. hierzu etwa die Textsammlung: Helmwart Hierdeis (Hg.): „Lieber Franz! Mein lieber Sohn!“ Antworten auf Franz Kafkas „Brief an den Vater“, Wien 1997. 
experimentellen Erprobung eines neues Verfahrens - zu prüfen, welche Facetten des ,Riesenbriefes' (Kafka) auf diese Weise in den Blick geraten und auf neue Weise interpretiert werden können. Und welche nicht. ${ }^{7}$

\section{Pädagogische Lektüren}

Klaus Mollenhauer platziert in seiner Studie Vergessene Zusammenhänge die einleitenden Sätze aus Kafkas Brief an exponierter Stelle: Weil er in den ersten Sätzen, die Franz Kafka an seinen Vater richtet, elementare Aufgaben der Pädagogik formuliert findet, setzt sein Entwurf einer Allgemeinen Pädagogik mit der Interpretation dieser Textpassage ein. Er liest somit Kafkas Brief als ein verlässliches Dokument, das für die erziehungswissenschaftliche Reflexion deshalb so wertvoll ist, weil es völlig frei von Euphemismen ist und ohne jenes „Fortschritts-Pathos“ auskommt ${ }^{8}$, das noch immer für zahlreiche pädagogische Texte charakteristisch ist. Fasziniert von der Genauigkeit der Beschreibung und der Präzision der Sprache, lenkt Mollenhauer den Blick auf drei zentrale Aufgaben der Pädagogik, die sich den Ausführungen Kafkas entnehmen lassen: Der Brief sensibilisiert demnach für die Einsicht, dass die Erziehung als eine nur begrenzt aufklärbare und unhintergehbare soziale Tatsache zu begreifen ist - als ein überaus komplizierter Sachverhalt also, der nicht nur nicht abgeschafft oder überwunden werden kann, sondern der sich auch einer vollständigen Durchdringung und Aufklärung entzieht. Darüber hinaus erinnert Kafkas unbestechlicher Blick, mit dem er die eigene Erziehung kritisch mustert, an die wichtige Aufgabe der selbstkritischen Prüfung, der sich Pädagogen nach Mollenhauer immer wieder unterziehen müssen: „Was darf, nach wahrhaftiger Prüfung der Erinnerung, als gerechtfertigt gelten?" Schließlich gilt ihm Kafkas unprätentiöser, fast schmuckloser Stil, der größtmöglicher Differenziertheit und höchster Genauigkeit verpflichtet ist, als Stachel im Fleisch der Pädagogik - den Brief an den Vater betrachtet er daher auch als ein sprachliches Kunstwerk, von dem sich die Erziehungswissen-

7 Pierre Bourdieu selbst warnt eindringlich vor der „Versuchung“, etwa das Verhältnis von Psychoanalyse und Soziologie als Alternative zu denken: „Die Soziologie hat nicht vor, die Erklärungsansätze der Psychoanalyse durch ihre eigenen zu ersetzen; sie versteht es lediglich, einige der Daten, die auch Gegenstand der Psychoanalyse sein können, auf andere Weise zu konstruieren, indem sie Aspekte der Realität genauer betrachtet, die die Psychoanalyse als sekundär oder unbedeutend aus der Analyse ausschließt oder die sie lediglich als Schutzschilde betrachtet, die es zu durchbrechen gilt, um zum Wesentlichen durchzudringen [...]. Diese Aspekte können wichtige Informationen im Hinblick auf Dinge enthalten, die auch die Psychoanalyse betrachtet.“ Pierre Bourdieu: „Widersprüche des Erbes“, in: ders. u.a., Das Elend der Welt. Zeugnisse und Diagnosen alltäglichen Leidens an der Gesellschaft, Konstanz 1997, S. 651-658, hier S. 657.

8 Vgl. K. Mollenhauer: Vergessene Zusammenhänge, S. 9.

9 Ebd., S. 12. 
schaft bei der Entwicklung einer geeigneten Theoriesprache anregen lassen könne.

Jürgen Oelkers begreift den Brief in seinem Aufsatz Väter und Söhne: Über Anklage, Missverständnis und den fehlenden Adressaten in der Erziehung weniger als authentisches Zeugnis, das einen objektiven Zugang zu dem ,tatsächlichen' Verhältnis zwischen Franz und Hermann Kafka ermöglicht; vielmehr interpretiert er ihn als den verzweifelten und um „Fairneß“ bemühten Versuch, das Erleben des „Erziehungsvater [s]“ zu rekonstruieren und dessen eigentümliche Macht zu verstehen, um sich auf diese Weise aus der Umklammerung durch den Vater zu lösen. ${ }^{10}$ Scheitern muss dieser Versuch, weil nach Oelkers Erziehung sich hier als ein gleichsam lebenslängliches Verhängnis darstellt, das als schicksalhafte Erfahrung ohnmächtig erlebt wird und folglich auch mit dem Erreichen der Volljährigkeit nicht beendet ist. Dabei lasse sich von Kafka lernen, dass es mitunter ausgesprochen schwierig ist, einen Adressaten zu identifizieren, der für die Erziehung einer Person verantwortlich zeichnet und folglich auch haftbar gemacht werden könnte. Erziehung werde daher höchst unzureichend gefasst, wenn sie als Verkettung intentionaler und zielgerichteter Handlungen eigener Art begriffen werde: Was Franz Kafka als Erziehung bezeichnet, ist daher - so Oelkers in einer vergleichenden Analyse mit den Kindheitserinnerungen Theodor Fontanes - keine „omnipotente Wirkkraft, sondern tritt differenziert auf als ungewollte, ja unschuldige, dennoch dauerhafte Prägung in der Folge einer Beziehungskonstellation, als beiläufige Hilfe und Unterstützung dessen, was ohnehin geschieht [...]. "11 Weil Kafka darum weiß, dass die Schuldzuweisung an die Adresse des Vaters lediglich Ausdruck einer zweifelhaften Attributionspraxis wäre, erhöht die analytische Durchdringung des Erziehungsprozesses noch die Last des Erzogenen - denn wo sich schuldhafte Verstrickungen ereignen, die sich keiner verantwortlichen Adresse zweifelsfrei zurechnen lassen, bleibt dem Opfer selbst die Möglichkeit der Anklage verwehrt.

Hans-Christoph Koller ist ebenfalls weniger an verlässlichen Auskünften über das Binnenleben der Familie Hermann Kafkas interessiert. Er nähert sich dem Brief aus einer bildungstheoretischen Perspektive und nimmt zu diesem Zweck eine Akzentverschiebung vor, die auch bei neueren literaturwissenschaftlichen Arbeiten zu Kafkas literarischen Texten ${ }^{12}$ zu beobachten ist: Statt das Augenmerk auf den Text zu richten - auf pädagogische Motive etwa oder auf Spuren, die der Erziehungsprozess hinterlassen hat -, konzentriert er sich

10 Jürgen Oelkers: „Väter und Söhne: Über Anklage, Mißverständnis und den fehlenden Adressaten in der Erziehung“, in: Neue Sammlung 38 (1998), S. 533-553, hier S. 535 und S. 537, Hervorhebung im Text.

11 Jürgen Oelkers: „Kindheit als Glück und als Geißel: Fontanes und Kafkas Erinnerungen im Vergleich“, in: ders., Die Herausforderung der Wirklichkeit durch das Subjekt. Literarische Reflexionen in pädagogischer Absicht, Weinheim, München 1985, S. 21-53, hier S. 52.

12 Vgl. hierzu etwa Detlef Kremer: Kafka. Die Erotik des Schreibens, Bodenheim 1998. 
in Negativität und Bildung. Eine bildungstheoretisch inspirierte Lektüre von Franz Kafkas „Brief an den Vater" auf das Abfassen des Textes selbst. ${ }^{13}$ Im Akt des Schreibens komme es - so Kollers zentrale These - zu einer weitreichenden Veränderung und Umarbeitung bedeutsamer Elemente des Selbstund Weltverhältnisses, die sich als Bildungsprozess begreifen lässt. ${ }^{14}$ Der Anlass für die komplizierte Arbeit an sich ist eine Paradoxie, in die sich Franz Kafka schon sehr früh verstrickt sieht und die ihn in seiner Entwicklung in besonderer Weise hemmt: Weil er die Selbständigkeit nur in Beziehung zu seinem Vater erringen kann, bleibt er selbst noch bei seinem Versuch der Ablösung auf diesen rückbezogen. Er sieht sich daher in einer ausweglosen, lähmenden Abhängigkeit seinem Vater gegenüber gefangen: „Ich war immerfort in Schande, entweder befolgte ich Deine Befehle, das war Schande, denn sie galten ja nur für mich; oder ich war trotzig, das war auch Schande, denn wie durfte ich Dir gegenüber trotzig sein, oder ich konnte nicht folgen, weil ich z.B. nicht Deine Kraft, nicht Deinen Appetit, nicht Deine Geschicklichkeit hatte, trotzdem Du es als etwas Selbstverständliches von mir verlangtest; das war allerdings die größte Schande.“ (B 128) ${ }^{15}$ Um sich dieser Abhängigkeitsbeziehung gegenüber jedoch nicht völlig wehrlos zu zeigen, entwickelt Kafka vier verschiedene Verfahren. Gemeinsam ist diesen Schreibtechniken, die von der ,Aufzeichnung' über die ,Übertreibung' und die ,Metaphorisierung' bis hin zur ,Modalisierung' reichen ${ }^{16}$, dass sie mit dem Ziel eingesetzt werden, die prägende Erfahrung des Vaters zu bearbeiten, in ein distanziertes Beobachterverhältnis zu diesem zu gelangen und das eigene Erleben einer befreienden Transformation zu unterziehen.

Auch wenn die skizzierten pädagogischen Lektüren von Franz Kafkas Brief an den Vater kein einheitliches Bild entwerfen und je spezifische Lesarten entwickeln, so stimmen sie doch darin überein, dass der Schlüssel zu den dargestellten Problemkreisen - der Entfremdung voneinander, dem wechselseitigen Unverständnis und den gegenseitigen Verletzungen - meist bei den Akteuren selbst gesucht wird: In der unheilvollen Konfrontation extrem unterschiedlicher Charaktere, die sich einander kaum einmal verständlich machen konnten, liege der eigentliche Grund dieser gleichsam lebenslänglichen Verfehlung von Vater und Sohn. Die Suche nach jenen sozialen Kräften, die zu dieser spannungsvollen Konstellation geführt haben, wird allerdings nur höchst selten betrieben. Bevor dies geschieht, gilt es sich freilich kurz des the-

13 Hans-Christoph Koller: „Negativität und Bildung. Eine bildungstheoretisch inspirierte Lektüre von Franz Kafkas ,Brief an den Vater““, erscheint in: SG, Beiheft der Zeitschrift für Pädagogik, 2005.

14 Vgl. Hans-Christoph Koller: Bildung und Widerstreit. Zur Struktur biographischer Bildungsprozesse in der (Post-) Moderne, München 1997.

15 Franz Kafka: Brief an den Vater. Faksimile. Herausgegeben und mit einem Nachwort versehen von Joachim Unseld, Frankfurt/Main 1994, S. 128. Diese Ausgabe wird direkt im Text mit der Sigle B zitiert.

16 Vgl. H.-Ch. Koller: Negativität und Bildung, S. 10-12. 
oretischen Instrumentariums zu versichern, das diese Spurensuche ermöglichen soll. ${ }^{17}$

\section{Pierre Bourdieus Theoriedes sozialen Raumes}

Pierre Bourdieus Entwurf einer Theorie des sozialen Raumes geht hervor aus dem Versuch, die komplizierte Beziehung zwischen sozialen Praktiken und gesellschaftlichen Strukturen zu klären, ohne sich dabei in jene Aporien zu verstricken, die für subjektivistische oder objektivistische Paradigmen charakteristisch sind. Statt etwa auf handlungstheoretische oder marxistische Ansätze zurückzugreifen, bemüht er sich, eine strikt relationale Analyse sozialer Phänomene zu entwickeln, die deren Bedeutung konsequent differenztheoretisch erklärt und damit allen essentialistischen Erklärungsmustern eine Absage erteilt $^{18}$ : In Anlehnung an Ernst Cassirers Unterscheidung zwischen, substantiellen' und ,funktionalistischen Begriffen ${ }^{619}$ entwickelt er eine theoretische Untersuchungsperspektive, die die Bedeutung, die einem Gegenstand, einer Handlung oder etwa einem Urteil beigemessen wird, immer in Abhängigkeit von der Gruppe der alternativen, nicht gewählten Gegenstände, Handlungen und Urteile bestimmt. Den Elementen der sozialen Welt wird daher jeder immanente oder absolute Wert abgesprochen. Entsprechend zustimmend zitiert Bourdieu den strukturalistischen Linguisten und Sprachhistoriker Emil Beveniste: „Sich unterscheiden und etwas bedeuten ist ein- und dasselbe“ ${ }^{20}$

Auch das Modell des sozialen Raumes konstruiert Bourdieu - strikt relational - über das Geflecht von Beziehungen, das zwischen dessen Elementen existiert und unterschiedliche Positionen definiert. Um nun die Verteilung der einzelnen Akteure und gesellschaftlichen Gruppen sowie die Zuordnung zu den differenten Positionen erklären zu können, ist es nach Bourdieu unverzichtbar, zwei wichtige Dimensionen des Kapitals zu berücksichtigen: Zum

17 Andere Anschlussmöglichkeiten an die Arbeiten Bourdieus habe ich skizziert in: Markus Rieger-Ladich, „Böse Blicke. Streifzüge durch das Feld der Erziehungswissenschaft mit Pierre Bourdieu“, in: Jan Masschelein/Wolfgang Nieke/Ludwig Pongratz (Hg.), Kritik der Pädagogik - Pädagogik als Kritik, Opladen 2004, S. 134-155.

18 Vgl. hierzu etwa Pierre Bourdieu: „Strukturalismus und soziologische Wissenschaftstheorie“, in: ders., Zur Soziologie der symbolischen Formen, Frankfurt/Main 1994, S. 7-41; Markus Rieger-Ladich: Mündigkeit als Pathosformel. Beobachtungen zur pädagogischen Semantik, Konstanz 2002, S. 297-314. Zu einer etwas anderen Einschätzung gelangen: Roland Lippuner/Julia Lossau: In der Raumfalle. Eine Kritik des spatial turn in den Sozialwissenschaften, in diesem Band.

19 Vgl. Ernst Cassirer: Substanzbegriff und Funktionsbegriff. Untersuchungen über die Grundfragen der Erkenntniskritik, Berlin 1923.

20 Pierre Bourdieu: „Sozialer Raum, symbolischer Raum“, in: ders., Praktische Vernunft, Frankfurt/Main 1998, S. 13-32, hier S. 22. 
einen ist das „Gesamtvolumen des Kapitals ${ }^{\text {“21 }}$ bedeutsam, das über die Addition des ökonomischen, des kulturellen und des sozialen Kapitals bestimmt wird. Hier wird folglich in Rechnung gestellt, in welchem Umfang etwa Vermögenswerte angehäuft, Bildungstitel erworben und Beziehungen zu jener Gruppe von gesellschaftlichen Entscheidungsträgern geknüpft wurden, die deren gewinnbringenden Einsatz versprechen. ${ }^{22}$ Zum anderen spielt die „Struktur dieses Kapitals" - also die relative Gewichtung der unterschiedlichen Kapitalsorten - eine nicht weniger wichtige Rolle. ${ }^{23}$ Dabei gilt ein besonderes Augenmerk dem ökonomischen und dem kulturellen Kapital, weil diese in den komplexen, hochgradig ausdifferenzierten Gesellschaften der westlichen Welt gegenwärtig dominieren und daher die Positionierung innerhalb des sozialen Raumes maßgeblich bestimmen.

Berücksichtigt man diese Konstruktionsprinzipien, wird deutlich, dass der soziale Raum hierarchisch verfasst ist: Er verdankt seine Struktur sowohl der Quantifizierung der unterschiedlichen Kapitalvolumina als auch den Beziehungen von Nähe und Distanz, die die einzelnen Akteure miteinander unterhalten. Auch wenn sich Bourdieu in diesem Punkt ausdrücklich von Karl Marx distanziert und dessen Vertrauen auf die Existenz realer sozialer Klassen wiederholt kritisiert ${ }^{24}$, arbeitet er doch heraus, dass es an den unterschiedlichsten Stellen innerhalb des sozialen Raumes regelmäßig zu charakteristischen Verdichtungen und typischen Clusterbildungen kommt. Weil zwischen jenen sozialen Akteuren, deren Kapital nicht nur vom Umfang her vergleichbar ist, sondern sich auch in der Gewichtung der einzelnen Kapitalsorten entspricht, zahlreiche ,Familienähnlichkeiten' - Übereinstimmungen in der Geschmacksbildung und der Wahl des Lebensstils etwa - beobachtet werden können, kommt es hier zur beiläufigen, kaum einmal gezielt betriebenen Ausbildung von Nähe und Vertrautheit stiftenden Affinitäten. ${ }^{25}$ Dieses Phänomen lässt sich freilich in allen Segmenten des sozialen Raumes beobachten - es kennzeichnet folglich nicht nur die gesellschaftlichen Eliten, die sowohl über hohes kulturelles als auch über hohes ökonomisches Kapital verfügen und daher die Spitzenpositionen inne haben, es lässt sich auch bei jenen Akteuren beobachten, denen es nicht gelungen ist, die beiden wichtigsten Kapitalsorten

21 Vgl. ebd., S. 18.

22 Vgl. zur Unterscheidung der Kapitalsorten: Pierre Bourdieu: „Ökonomisches Kapital - Kulturelles Kapital - Soziales Kapital“, in: ders., Die verborgenen Mechanismen der Macht. Schriften zu Politik \& Kultur 1, hg. v. Margareta Steinrücke, Hamburg 1997, S. 49-79.

23 Vgl. ebd., S. 18.

24 Vgl. Pierre Bourdieu: „Sozialer Raum und „Klassen““, in: ders., Sozialer Raum und ,Klassen“. Leçon sur la leçon. Zwei Vorlesungen, Frankfurt/Main 1995, S. 7-46.

25 Deutlich wird dies etwa bei der Interpretation einer Fotographie durch Gruppen, die sich hinsichtlich des kulturellen Kapitals deutlich voneinander unterscheiden. Vgl. Burkhard Michel/Jürgen Wittpoth: Substanzielle und strukturelle Dimensionen kulturellen Kapitals. Habitusspezifische Sinnbildungsprozesse bei der Rezeption von Fotografien, in diesem Band. 
in ausreichendem Maße zu akkumulieren und die daher nicht selten auf die sozialen Sicherungssysteme angewiesen sind, weil sie sich innerhalb der gesellschaftlichen Verteilungskämpfe als kaum konkurrenzfähig erwiesen haben. $^{26}$

Um nun die stillschweigenden Übereinkünfte zu erklären, die sich zwischen eng benachbarten Positionen innerhalb des sozialen Raumes feststellen lassen, greift Bourdieu auf den lateinischen Begriff habitus zurück und verleiht ihm eine besondere Prägung: Der Habitus bezeichnet danach jene charakteristische Konfiguration von Handlungs-, Erfahrungs- und Bewertungsmustern, die eine Person in Abhängigkeit von den Möglichkeiten ausbildet, die ihr die eigene Position innerhalb des sozialen Raumes eröffnet. Als vereinheitlichendes Prinzip erzeugt er daher nicht nur einen hochentwickelten „Sinn für Grenzen“،27, der zuverlässig regelt, was einer Person ,unmöglich“ ist, selbst wenn die materiellen Voraussetzungen erfüllt sind und die Bedingungen daher als überaus günstig erscheinen mögen, sondern er verweist auch auf die Vergangenheit und damit auf die Bedeutung der sozialen Herkunft: „Als Produkt der Geschichte produziert der Habitus individuelle und kollektive Praktiken, also Geschichte, nach den von der Geschichte erzeugten Schemata; er gewährleistet die aktive Präsenz früherer Erfahrungen, die sich in jedem Organismus in Gestalt von Wahrnehmungs-, Denk- und Handlungsschemata niederschlagen und die Übereinstimmung und Konstantheit der Praktiken im Zeitverlauf viel sicherer als alle formalen Regeln und expliziten Normen zu gewährleisten suchen. ${ }^{\prime 28}$

Gerade weil dies den Eindruck einer leibnizschen prästabilisierten Harmonie nahe legen könnte, die aus der je individuellen Anerkennung des eigenen Ranges und der daran geknüpften Möglichkeiten hervorgeht, gilt es festzuhalten, dass der soziale Raum keineswegs statisch verfasst ist und sich über die immer gleichen Mechanismen reproduziert. Vielmehr weist Bourdieu nachdrücklich darauf hin, dass für das Modell des sozialen Raumes eine besondere Dynamik charakteristisch ist: Weil sich beobachten lässt, dass eine Vielzahl der sozialen Akteure - einzelne Individuen oder gesellschaftliche Gruppen größte Anstrengungen unternehmen, um ihre soziale Position zu verbessern, muss statt dessen mit fortwährenden gesellschaftlichen Verteilungskämpfen gerechnet werden. Dabei wird deutlich, dass immer dann, wenn die existierende gesellschaftliche Ordnung ihre Aura der Unangreifbarkeit verliert und die eingespielten Verrechnungskurse für die unterschiedlichen Kapitalsorten ihren Anschein der Notwendigkeit einbüßen - wenn also die symbolische Herrschaft als jene Kraft enttarnt wird, die weitgehend unbemerkt und der

26 Diese soziale Gruppe steht im Zentrum einer großen Gemeinschaftsstudie französischer Sozialwissenschaftler, die von Pierre Bourdieu geleitet wurde: P. Bourdieu u. a.: Das Elend der Welt, a.a.O.

27 Pierre Bourdieu: Die feinen Unterschiede. Kritik der gesellschaftlichen Urteilskraft, Frankfurt/Main 1992, S. 734. Hervorhebung im Text.

28 Pierre Bourdieu: Sozialer Sinn. Kritik der theoretischen Vernunft, Frankfurt/ Main 1993, S. 101. 
Kritik entzogen die Reproduktion der bestehenden Verhältnisse zu legitimieren unternimmt ${ }^{29}$-, diejenigen ihre Anstrengungen noch weiter intensivieren, die vom Status quo benachteiligt werden. Vielleicht am anschaulichsten hat Bourdieu die besondere Dynamik, der soziale Räume gegenwärtig unterliegen, in jener Untersuchung herausgearbeitet, die ihn auch in Deutschland in kürzester Zeit einem breiten Publikum bekannt gemacht hat. In La distinction, das in der deutschen Übersetzung mit dem irreführenden Titel Die feinen Unterschiede versehen wurde, beschreibt er die französische Gesellschaft der 1970er Jahre als einen vibrierenden Körper, der permanenten Erschütterungen ausgesetzt ist: Zwischen den herrschenden Eliten, die ein gesteigertes Interesse daran haben, die existierende Verteilung ökonomischen, kulturellen und sozialen Kapitals zu verteidigen und als gerechtfertigt erscheinen zu lassen, und den Gruppen depravierter Akteure, die kaum noch in der Lage sind, in die Verteilungskämpfe einzugreifen, sind die Fraktionen des (Klein-)Bürgertums angesiedelt, die ihre Hoffnungen, die eigene Position zu verbessern, an das Bildungswesen knüpfen und auf diesem Wege ihren Mangel an ökonomischem Kapital zu kompensieren versuchen. ${ }^{30}$

Innerhalb dieser verbissen geführten Kämpfe um Aufstiegschancen und Positionsverbesserungen erweist sich auch gegenwärtig die Familie als einer der erfolgreichsten sozialen Akteure. Obwohl sich die Reproduktionsmechanismen in der Folge der Autonomisierung der sozialen Felder und der damit einhergehenden größeren Unabhängigkeit des Bildungswesens verändert haben - die Vererbung ökonomischen Kapitals verliert in den funktional differenzierten Gesellschaften gegenüber der Akkumulation kulturellen Kapitals zweifellos an Bedeutung -, bleibt die Familie eine höchst erfolgreiche Agentur bei der Zuweisung sozialer Chancen. Auch unter den veränderten Bedingungen gelingt es ihr, die eigenen Mitglieder aussichtsreich zu platzieren: „Die Familie spielt [...] für die Reproduktion der Struktur des sozialen Raums und der gesellschaftlichen Verhältnisse [...] eine entscheidende Rolle. Sie ist einer der bevorzugten Orte der Akkumulation von Kapital aller Sorten und seiner Weitergabe von Generation zu Generation: Sie wahrt ihre Einheit für die Weitergabe und durch die Weitergabe [...]. Sie ist das wichtigste ,Subjekt ${ }^{6}$ der Reproduktionsstrategien." ${ }^{\text {31 }}$ Bemerkenswert ist dieser Sachverhalt nicht zuletzt deshalb, weil er mit dem Selbstverständnis demokratischer Gesellschaften kaum zu vereinbaren ist: Obwohl diese sich dem Prinzip der Chancengleichheit verschrieben haben und um die Etablierung eines Bildungswesens bemühen, das die Herkunft der Schüler nicht länger prämiert, behauptet

29 Vgl. hierzu Pierre Bourdieu: Was heißt Sprechen? Die Ökonomie des sprachlichen Tausches, hg. v. Georg Kremnitz, Wien 1990.

30 Vgl. P. Bourdieu: Die feinen Unterschiede, bes. S. 195-276 und S. 500-584.

31 Pierre Bourdieu: Familiensinn, in: ders., Praktische Vernunft, S. 126-136, hier S. 132. 
sich die Familie als zentrale Adresse für die Verteilung gesellschaftlicher Chancen. $^{32}$

\section{Franz Kafkas Brief an den Vater}

Wirft man nun einen solcherart instrumentierten Blick auf Franz Kafkas Brief an den Vater, geraten nicht wenige Themen und Gegenstände, die von Literatur- oder Erziehungswissenschaftlern diskutiert werden, in ein etwas verändertes Licht. Wie sich die Perspektiven verschieben, wenn man Bourdieus Überlegungen zur Struktur und Dynamik des sozialen Raumes systematisch berücksichtigt, lässt sich an einer Begebenheit andeuten, die innerhalb der Literatur zu Kafkas Brief kaum einmal zum Gegenstand einer genaueren Betrachtung wird, die sich vielleicht aber doch in heuristischer Hinsicht als aufschlussreich erweisen könnte. Es handelt sich dabei um Hermann Kafkas abfälligen Kommentar über einen Schauspieler, den sein Sohn wahrscheinlich über Max Brod kennen gelernt hatte und der in den Jahren 1911 und $1912 \mathrm{zu}$ einem Freund geworden war. ${ }^{33}$ Im Brief wird dieser Vorfall, der zum Zeitpunkt des Abfassens bereits acht Jahre zurückliegt, wie folgt kommentiert: „Es genügte, dass ich an einem Menschen ein wenig Interesse hatte - es geschah ja infolge meines Wesens nicht sehr oft - dass Du ohne jede Rücksicht auf mein Gefühl und ohne Achtung vor meinem Urteil mit Beschimpfung, Verläumdung, Entwürdigung dreinfuhrst. Unschuldige, kindliche Menschen wie z.B. der jiddische Schauspieler Löwy mussten das büssen. Ohne ihn zu kennen, verglichst Du ihn in einer schrecklichen Weise, die ich schon vergessen habe, mit Ungeziefer und wie so oft für Leute, die mir lieb waren, hattest Du automatisch das Sprichwort von den Hunden und Flöhen bei der Hand.“ (B 125)

Was hier als rücksichtsloser, wenig einfühlsamer Umgang Hermann Kafkas mit einer jener Personen erscheint, zu denen Franz Kafka freundschaftliche Beziehungen pflegt, erweist sich als ungleich komplexere Reaktion, rekonstruiert man nun den sozialen Raum, innerhalb dessen es zu dieser Begegnung kommt: Jizchak Löwy musste für Hermann Kafka genau jene Fraktion des Judentums verkörpern, die er bei seinem energisch betriebenen, mitunter auch entbehrungsreichen sozialen Aufstieg endlich hinter sich gelassen hatte und die in Prag sowohl von den herrschenden Eliten als auch den assimilierten Juden mit Geringschätzung behandelt wurde. Der in Warschau geborene Jude, der die russische Staatsbürgerschaft besaß und fortwährend in größte finanzielle Nöte geriet, war Mitglied eines Ensembles, das sich Original-Polnisch-

32 Vgl. Markus Rieger-Ladich: „Unbequeme Zaungäste? Pierre Bourdieu und Niklas Luhmann beobachten die Familie und irritieren die Erziehungswissenschaft", in: Vierteljahrsschrift für wissenschaftliche Pädagogik 80 (2004), S. 85-102.

33 Vgl. zu den historisch-biographischen Hintergründen im Folgenden die vorzügliche Kafka-Biographie: Reiner Stach: Franz Kafka. Die Jahre der Entscheidungen, Frankfurt/Main 2002, bes. S. 46-65. 
jüdische-Gesellschaft nannte und von Herbst 1911 bis zur Mitte des Januar 1912 in Prag gastierte. Weil sich der vornehme Festsaal des Hotel Central, der vom Prager Bürgertum besucht wurde, nicht als der passende Rahmen erwies und sich keine Besucher einfanden, spielte die ostjüdische Theatergruppe schon sehr bald in einem kleinen Nebenraum des etwas heruntergekommenen Café Savoy. Hier kam es zur Aufführung von Stücken, die kaum geeignet waren, in den Feuilletons der beiden deutschsprachigen Tageszeitungen auf positive Resonanz zu stoßen, und denen von der Mehrzahl des gebildeten Prager Bürgertums wahrscheinlich der Kunstcharakter vollständig abgesprochen wurde. Franz Kafka hat diese offensichtlich überaus dynamischen und expressiven Aufführungen, die von - so sein Tagebucheintrag vom 26. Oktober 1911 - „Peitschen, Wegreißen, Schlagen, Achselnbeklopfen, Ohnmächtigwerden, Halsabschneiden, Hinken, Tanzen in russischen Stulpenstiefeln, Tanzen mit gehobenen Frauenröcken, Wälzen auf dem Kanapee“" geprägt waren ${ }^{34}$, als authentischen Ausdruck jüdischer Volkskultur interpretiert und überaus geschätzt. Für Hermann Kafka freilich, der selbst dann nicht zu einer Aufführung erscheint, als sein Sohn einen Soloauftritt des Freundes organisiert und zu diesem Zweck sogar eine kurze einführende Rede hält, musste dieser mittellose, schlecht gekleidete, jiddisch sprechende, der deutschen Schriftsprache kaum mächtige, offensichtlich erfolglose Schauspieler als erschreckend präziser Gegenentwurf dessen wirken, was er für seinen eigenen Sohn erhoffte und anzubahnen sich bemühte.

Um dieses - aus der Perspektive Franz Kafkas - harsche und demütigende Urteil zu erklären, ist es unverzichtbar, das gesellschaftliche Kräftefeld zu skizzieren, das zu jener Zeit in Prag herrscht. Hermann Kafka ${ }^{35}$ zählt zu jener Generation böhmischer Dorfjuden, für die die Aufhebung des Familiantengesetzes im Jahr 1849, das sie in ihren bürgerlichen Rechten stark eingeschränkt hatte $^{36}$, zu einem Signal wurde, nun den sozialen Aufstieg unter Aufbietung aller Kräfte zu betreiben. Die Strategie, die er dabei wählt, scheint vorgezeichnet: Ohne über nennenswerte ökonomische Besitztümer oder gar kulturelles Kapital zu verfügen - der Besuch der jüdischen Schule von Osek, in der Deutsch als Unterrichtssprache gepflegt wurde, endete bereits nach der Bar Mizwa -, bleibt ihm nur die Möglichkeit, verwandtschaftliche Kontakte zu nutzen und dieses Beziehungsnetz zu pflegen. Als er allerdings im Jahr 1882 Julie Löwy, die aus einer mittelständischen jüdischen Familie stammt, heiratet $^{37}$, verfügt er mit der Mitgift endlich über jene finanziellen Mittel, die ihm die Gründung eines eigenen Geschäfts ermöglichen. Allerdings kann das $G a$ lanteriewaren-Geschäft Hermann Kafka kaum als besonders krisensicher gelten, weil die angebotenen Waren - Handschuhe, Spazierstöcke, Tücher oder

34 Zitiert nach: ebd., S. 48f.

35 Vgl. Alena Wagnerowá: „Im Hauptquartier des Lärms“. Die Familie Kafka aus Prag, Köln 1997.

36 Vgl. ebd., S. 21-36.

37 Um Missverständnisse zu vermeiden, sei angemerkt, dass Julie Löwy nicht mit Jizchak Löwy verwandt ist. 
etwa ausgesuchte Unterwäsche - zweifellos zu den Gegenständen zählen, die in Zeiten finanzieller Not als durchaus verzichtbar galten. Um so bemerkenswerter ist der ökonomische Erfolg, der schließlich dazu führt, dass zwanzig Jahre nach der Geschäftsgründung und nach einigen Umzügen Ladenräume im Kinsky-Palais am Altstädter Ring angemietet werden können. Der KafkaBiograph Reiner Stach hat diesen Triumph Hermann Kafkas mit feinem Gespür für das Auseinanderklaffen der Bewegungen im sozialen und im physischen Raum treffend festgehalten: „Der Umzug dorthin führte eigentlich nur um die Ecke, kaum hundert Meter waren zu überbrücken. Doch das Ladenschild am zentralen Platz der Prager Altstadt bedeutete einen Zuwachs an symbolischem Kapital, das sich bald auch in klingender Münze auszahlte.“38

Hermann Kafka war daher ein durchaus typischer Vertreter des jüdischen Mittelstands in Prag, der zwar erfolgreich ökonomisches Kapital akkumuliert hatte, der aber gleichwohl sehr genau um seinen prekären Status wusste: Zwischen der zahlenmäßig großen Bevölkerungsgruppe der Tschechen, die nur über geringes Kapital verfügte, und der zahlenmäßig kleinen Gruppe der Deutschen, die unbestritten die herrschende Gesellschaftsschicht darstellte und die höchsten Positionen bekleidete, waren die Juden, unter denen viele als Geschäftsleute durchaus beachtlichen Erfolg hatten, in einer heiklen Position. Weil sie sich in Fragen des kulturellen Lebens fast bedingungslos an dem Lebensstil der kleinen deutschen Elite orientierten, bemühten sie sich - nicht selten: übereifrig -, jene Spuren zu tilgen, die noch an ihre einfache, meist ländliche Herkunft erinnerten. Für Hermann Kafka galt dies wahrscheinlich in besonderer Weise. Alena Wagnerowá hat ihn denn auch überzeugend als sozialen Aufsteiger porträtiert, der in der böhmischen Metropole bald an seine Grenzen stieß: „Den Landjuden hatte Hermann Kafka zwar abgestreift, die selbstverständlichen Standards eines Angehörigen des Mittelstandes hat er aber, mit Ausnahme der materiellen Voraussetzungen, nie ganz erreicht. Kulturell blieb Hermann Kafka zeitlebens ein Mensch im Niemandsland.“39

Es ist aus diesem Grund gar nicht einfach zu bestimmen, welche der unzähligen Stigmata Jizchak Löwys auf Hermann Kafka am abstoßendsten wirkten. Vielleicht fällt dabei noch stärker als der ökonomische Misserfolg, die zweifelhafte Berufswahl und die mangelnde gesellschaftliche Reputation, die Herkunft und die Sprache ins Gewicht. Die Ostjuden, zu denen Löwy zählte, galten - bezeichnenderweise gerade aus der Perspektive der Westjuden - nicht nur als ,unrein“40 und kaum gesellschaftsfähig, sie sprachen darüber hinaus auch noch demonstrativ Jiddisch - und damit einen bloßen ,Jargon " - und versäumten es auf diese Weise, über die Kultivierung des Deutschen ganz gezielt den Anschluss an die gesellschaftlichen Eliten zu suchen und die Sprache als eines der wichtigsten Medien des sozialen Aufstiegs zu begreifen. Marthe Robert hat den erheblichen Distinktionsgewinn, der sich mit der deutschen Spra-

38 R. Stach: Franz Kafka, S. 12.

39 A. Wagnerowá: Im Hauptquartier des Lärms, S. 60f.

40 R. Stach: Franz Kafka, S. 60. 
che zu dieser Zeit noch verbuchen lie $\beta^{41}$, anschaulich geschildert: „Auf diese Sprache [die deutsche; MRL] bauen die jüdischen Väter also am häufigsten, auch wenn sie bei ihren Geschäften oder im privaten Kreis weiterhin tschechisch und jiddisch sprechen (selbstverständlich ist das Jiddische völlig aus der Erziehung verbannt, es gibt keine anständige jüdische Familie, in der es nicht üblich ist, es zu verachten, in diesem wie in vielen anderen Punkten bildet die Familie Kafka keine Ausnahme).“42

Berücksichtigt man diese Tatsachen, erscheint die Reaktion von Hermann Kafka auf Jizchak Löwy nicht länger als Ausdruck einer besonders ausgeprägten Unbeherrschtheit oder Rücksichtslosigkeit. Statt dessen wird deutlich, dass die Wahrnehmung des jiddischen Schauspielers in engem Zusammenhang mit der Position steht, die dessen Betrachter innerhalb des sozialen Raumes einnehmen: Hermann Kafka fühlt sich durch den verarmten und verwahrlosten Juden aus der Provinz offensichtlich nicht nur an jene Verhältnisse erinnert, die er selbst erst durch extreme Anstrengungen erfolgreich hat überwinden können, sondern er scheint darüber hinaus zu befürchten, dass sich sein Sohn durch den zweifelhaften Freund ,infizieren' und den mühsam erreichten gesellschaftlichen Status leichtfertig verspielen könnte. Dies ließe sich als der biographische Hintergrund für das Bemühen des erniedrigenden Sprichworts vermuten, durch das Löwy - nach der Bezeichnung als Ungeziefer - noch weiter gedemütigt wird: „Wer mit Hunden zu Bett geht, steht mit Flöhen auf.“433 Franz Kafka hingegen scheint völlig frei zu sein von den typischen Ängsten des ambitionierten Aufsteigers, der von der Sorge um den Verlust des Erreichten getrieben wird und daher größten Wert darauf legt, die gesellschaftlichen Konventionen peinlich genau zu wahren und sich den Erwartungen gegenüber konform zu verhalten, die an den eigenen Stand gestellt werden: Als Schriftsteller, der gleichsam nebenbei auch als promovierter Jurist beruflich recht erfolgreich ist, begeistert er sich für den unkonventionellen Charakter und die ungekünstelten Momente der jiddischen Theaterstücke und schätzt dabei an seinem Freund Jizchak Löwy genau jene Charakterzüge, die diesen seinem Vater höchst suspekt und wenig vertrauenswürdig erscheinen lassen müssen. ${ }^{44}$

Im Zentrum des Konflikts zwischen Franz Kafka und Hermann Kafka steht daher - liest man den Brief an den Vater vor der Folie der Arbeiten Pierre Bourdieus - die große Entfernung, die diese innerhalb des sozialen Raumes voneinander trennt: Der Habitus des Aufsteigers, der als jüdischer Geschäftsmann beachtlichen ökonomischen Erfolg errungen hat, der freilich kaum kulturelles Kapital akkumuliert hat und um seine gefährdete gesell-

41 Die Konjunkturen der deutschen und der tschechischen Sprache verzeichnet: A. Wagnerová: Im Hauptquartier des Lärms, S. 174.

42 Marthe Robert: Einsam wie Franz Kafka. Aus dem Französischen von Eva Michel-Moldenhauer, Frankfurt/Main 1985, S. 36f.

43 Vgl. H.-Ch. Koller: Negativität und Bildung, S. 10.

44 Vgl. hierzu auch Ernst Pawel: Das Leben Franz Kafkas, München, Wien 1986, S. 273-284. 
schaftliche Stellung weiß, trifft auf den Habitus des Schriftstellers und Intellektuellen, der in seiner beruflichen Tätigkeit als Jurist keine Befriedigung erfährt und der Welt der Ökonomie völlig fremd gegenübersteht. Diese Konstellation, die ohnehin als überaus spannungsreich gelten muss und auch von Julie Löwy kaum entschärft werden kann, spitzt sich noch zu durch die Überlagerung von sozialem und physischem Raum. ${ }^{45}$ Weil Franz Kafka, der der (Geschäfts-)Welt seines Vaters meist ohnehin nur mit äußerster Distanz begegnet, bis zu seinem 31. Lebensjahr im Haushalt seiner Eltern lebt, kommt es zur Ausprägung extremer Formen von Fremdheit auf engstem Raum. Den unterschiedlichen Positionen, die sie innerhalb des sozialen Raumes bekleiden, fehlt daher eine Korrespondenz innerhalb des physischen Raumes - in der Folge bewohnen Vater und Sohn zwar dieselbe Wohnung, aber es kommt doch kaum einmal zu einer Begegnung, die von gegenseitigem Verständnis geprägt wäre. Auf schmerzhafte Weise deutlich wird dies etwa, als Hermann Kafka im Herbst 1911 - in jener Zeit also, in der sich Franz Kafka mit Jizchak Löwy anfreundet - seinen Sohn auf erheblichen Druck hin als stillen Teilhaber der Prager Asbestwerke Hermann und Co. gewinnt, damit auf diese Weise nicht nur ein beträchtlicher Teil der Mitgift an die Tochter Gabriele in den Händen der Familie bleibt, sondern dieser auch die - mitunter etwas risikofreudigen - Entscheidungen des Schwiegersohns kontrolliert. ${ }^{46}$ In der Folge dieser verhängnisvollen Zusage, die Franz Kafka sehr schnell bereut, weil sie ihm die Konzentration auf das Schreiben fast unmöglich macht, und die ihn schließlich sogar mit Selbstmordgedanken spielen lässt, kommt es immer wieder zu ,absurden Familienszene[n] “, die die enorme Distanz zwischen den unterschiedlichen Habitus und die daraus resultierende wechselseitige Fremdheit eindrucksvoll demonstrieren: „Während im Wohnzimmer Hermann Kafka und Karl Hermann, Schwiegervater und Schwiegersohn, um die Fortexistenz der ,Prager Asbestwerke' kämpften, lag nebenan, hinter verschlossener Tür, der verantwortliche „Teilhaber“ ausgestreckt auf dem Kanapee und langweilte sich. “47

Franz Kafkas Brief an den Vater erscheint in dieser Perspektive als das peinlich genaue Protokoll zweier höchst unterschiedlicher Habitus, das kaum eine wichtige Dimension ausspart: Mit gleichsam schmerzhafter Lust an der kontrastiven Gegenüberstellung und größter Präzision schildert Franz Kafka etwa die Unterschiede bezüglich der Körperlichkeit, der Essgewohnheiten oder des Sprachgebrauchs. Stellt man dabei in Rechnung, dass - wie Bourdieu bemerkt hat - in der „Körperhaltung [...] das umfassende Verhältnis zur sozialen Welt zum Ausdruck" kommt ${ }^{48}$, gewinnen Kafkas Beobachtungen zur unterschiedlichen Körperlichkeit noch an Bedeutung. Zu Beginn des Briefes hält er diesbezüglich fest: „Ich war ja schon niedergedrückt durch Deine blosse

45 Vgl. hierzu aus bildungstheoretischer Perspektive Karin Priem: Pädagogische Räume - Räume der Pädagogik. Ein Versuch über das Dickicht, in diesem Band.

46 Vgl. R. Stach: Franz Kafka, S. 23-29.

47 Ebd. S. 139.

48 P. Bourdieu: Die feinen Unterschiede, S. 309. 
Körperlichkeit. Ich erinnere mich z.B. daran, wie wir uns öfters zusammen in einer Kabine auszogen. Ich mager, schwach, schmal. Du stark, gross, breit.“ (B 122) Dass sich diese Wahrnehmung auch nicht durch einen Hinweis auf den bloßen Altersunterschied entschärfen lässt, wird deutlich, wenn man nun auch noch die Ess- und Ernährungsgewohnheiten berücksichtigt: Auch hier bestechen Kafkas Beobachtungen durch die Genauigkeit, mit der sie zwei unterschiedliche Habitus beschreiben. Dabei liegt in diesem Fall das Augenmerk weniger auf der Frage der Gerechtigkeit, die Jürgen Oelkers aufgeworfen hat und die um die Frage kreist, weshalb der Vater sich an jene Regeln nicht gebunden sieht, die er als für alle verbindlich erklärt hat ${ }^{49}$, vielmehr gilt das Interesse den stark voneinander abweichenden Essgewohnheiten Hermann und Franz Kafkas, die einen unterschiedlich verfeinerten Lebensstil verraten. Das Verhalten des Vaters bei Tisch wird im Brief wie folgt beschrieben: „Weil Du entsprechend Deinem kräftigen Hunger und Deiner besonderen Vorliebe alles schnell, heiss und in großen Bissen gegessen hast, musste sich das Kind beeilen, düstere Stille war bei Tisch, unterbrochen von Ermahnungen: ,zuerst iss, dann sprich' oder ,schneller, schneller, schneller' oder ,siehst Du, ich habe schon längst aufgegessen'. [...] Bei Tisch durfte man sich nur mit Essen beschäftigen, Du aber putztest und schnittest Dir die Nägel, spitztest Bleistifte, reinigtest Dir mit dem Zahnstocher die Ohren.“ (B 127) Der Kontrast zu der Ernährung und den Essgewohnheiten Franz Kafkas lässt sich kaum überbieten, folgt man den Schilderungen Stachs, der den familiären Alltag genau rekonstruiert hat: „Erst gegen 21.30 Uhr wurde die letzte Mahlzeit eingenommen, zumeist wohl die ,Reste" vom Mittag, während Kafka, ein ebenso unbelehrbarer wie anspruchsvoller Vegetarier, unter den verächtlichen Blicken seines Vaters ein ganzes Sortiment von Tellern und Schüsselchen um sich aufbaute, wahlweise mit Joghurt, Nüssen, Kastanien, Datteln, Feigen, Trauben, Mandeln, Rosinen, Bananen, Orangen oder sonstigem teurem Obst, dazu ein wenig Vollkornbrot. “50

Es verwundert nun kaum, dass sich die Spannungen noch verstärken, als sich abzeichnet, dass Franz Kafka, der in seinem Brief bereitwillig einräumt,

49 Vgl. Jürgen Oelkers: „Vater? Vater!“, in: H. Hierdeis (Hg.), „Lieber Franz! Mein lieber Sohn!“, S. 205-216.

50 R. Stach: Franz Kafka, S. 13. Bourdieu hat den Zusammenhang zwischen Körper, Geschmack und sozialer Klasse in Die feinen Unterschiede präzise herausgearbeitet: „Der Geschmack: als Natur gewordene, d.h. inkorporierte Kultur, Körper gewordene Klasse, trägt er bei zur Erstellung des ,Klassenkörpers“; als inkorporiertes, jedwede Form der Inkorporation bestimmendes Klassifikationsprinzip wählt er aus und modifiziert er, was der Körper physiologisch wie psychologisch aufnimmt, verdaut und assimiliert, woraus folgt, daß der Körper die unwiderlegbarste Objektivierung des Klassengeschmacks darstellt, diesen vielfältig zum Ausdruck bringt [...]. In der Tat erweist sich über kulinarische Vorlieben, die über die gesellschaftlichen Bedingungen, unter denen sie entstanden sind, hinaus Bestand haben können [...], und natürlich über den Gebrauch des Körpers im Arbeitsprozeß wie in der Freizeit die klassenspezifische Verteilung der körperlichen Eigenschaften." (P. Bourdieu: Die feinen Unterschiede, S. 307.) 
dass er nicht über jenen „Kafka'schen Lebens-, Geschäfts-, Eroberungswillen“ verfügt (B 118), der Hermann Kafka auszeichnet, kaum geeignet ist, das väterliche Erbe anzutreten und zu einem späteren Zeitpunkt die Leitung des Familienbetriebes zu übernehmen. Er ließ aus der Sicht seines Vaters nicht nur jede Affinität zur Geschäftswelt schmerzlich vermissen und beschränkte seinen beruflichen Ehrgeiz auf die ökonomisch wenig einträgliche Laufbahn eines Beamten der Arbeiter-Unfall-Versicherungs-Anstalt; er zeigte sich darüber hinaus auch noch allen wohlmeinenden ,Bekehrungsversuchen' gegenüber erstaunlich resistent. Stellt man nun in Rechnung, dass die gesellschaftliche Stellung der Familie Hermann Kafkas keineswegs als völlig gesichert gelten konnte und die Frage der Übergabe des Erbes ohnehin in besonderer Weise symbolisch aufgeladen ist, wird deutlich, wie konfliktträchtig die Beziehung zwischen Vater und Sohn in dieser Phase ist. Nach Bourdieu sind dabei die Rollen stets eindeutig verteilt: Der Vater gilt ihm innerhalb der Familie als der „Träger und Werkzeug eines ,Projekts “ [...], das in die ererbten Dispositionen eingeschrieben ist und unbewusst, in und durch die Wesensart des Vaters, sowie explizit, in Form einer erzieherischen Aktivität, die auf die Sicherung des Fortbestands der Abstammungslinie ausgerichtet ist (also dessen, was in manchen Traditionen ,das Haus' genannt wird), weitergegeben wird." Auch die Aufgabe des Sohnes ist klar definiert: „Erbe zu sein bedeutet, diese immanenten Dispositionen zu übertragen, das Streben nach Fortdauer (conatus) zu befriedigen und sich bereitwillig zum gehorsamen Werkzeug dieses Reproduktions-,Projekts‘ zu machen. ${ }^{\text {“51 }}$ Dass sich diese Konstellation zwischen Vater und Sohn in einer Familie, die ihren Wohlstand einem Geschäft verdankt, das jener gegründet und stetig vergrößert hat, noch verschärft, erscheint somit wenig überraschend und fast zwangsläufig. Die „,Gespaltenheit` des Habitus" ist daher - dies hat Eva Barlösius im Rückgriff auf neuere Studien Bourdieus überzeugend herausgearbeitet - eine direkte Folge der beschriebenen Krise der „familialen Reproduktion““52, die bei der Familie Hermann Kafkas von den beteiligten Akteuren offensichtlich als besonders bedrohlich erlebt wurde. Es kommt zu diesem Phänomen nicht nur in jenen Fällen, in denen gesellschaftliche Umbrüche dazu führen, dass die Passung zwischen tatsächlichen Chancen und verfolgten Zielen, die in der Regel über den Habitus gewährleistet wird, verloren geht, sondern eben auch dann, wenn die ,jüngere Generation familial einen Habitus aufgeprägt bekommen hat, der nicht auf ihre reale Lage abgestimmt ist, und dies permanent das Gefühl des Verlustes in ihnen erzeugt. “"

Es spricht nun sowohl für Kafkas ausgeprägte Sensibilität als auch für die Redlichkeit der Auseinandersetzung mit seinem Vater, dass er diesen nicht

51 Pierre Bourdieu: „Widersprüche des Erbes“, in: ders. u. a., Das Elend der Welt, S. 651-658, hier S. 652.

52 Eva Barlösius: „,Das Elend der Welt‘. Bourdieus Modell für die ,Pluralität der Perspektiven“ und seine Gegenwartsdiagnose über die ,neoliberale Invasion“", in: BIOS 12 (1999), S. 3-27, hier S. 22.

53 Ebd. 
verantwortlich macht für die offensichtlich von beiden schmerzhaft erlebte „Entfremdung“; vielmehr erklärt er ausdrücklich, dass sie beide an dieser „gänzlich schuldlos“" seien. (B 117) Offensichtlich ahnt Franz Kafka, der seinem Vater schon bezüglich der eigenen Berufswahl attestiert, dass er hier wohl der „massgebenden allgemeinen Söhnebehandlung des jüdischen Mittelstandes" gefolgt sei (B 161), dass der Konflikt eben auch auf das Wirken sozialer Kräfte zurückgeht, die nicht einfach Individuen und deren Handlungen zugerechnet werden können. Im ungefähren Wissen um schuldhafte Verstrickungen, die keine verantwortlichen Subjekte kennen, spricht er den eigenen Vater in seiner kunstvollen Anklageschrift denn auch frei und räumt ein, dass diesen keine Schuld treffe: „Du wirktest so auf mich, wie Du wirken

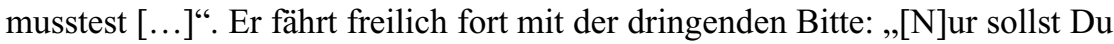
aufhören, es für eine besondere Bosheit meinerseits zu halten, dass ich dieser Wirkung erlegen bin.“ (B 119)

Der Grundton der Verzweiflung, der Franz Kafkas Brief an den Vater prägt, lässt sich daher womöglich auch als Eingeständnis interpretieren, dass der Versuch, sich dieser unheilvollen Wirkung dauerhaft zu entziehen, zum Scheitern verurteilt ist. Nachdem bereits seine früheren Versuche, sich vom Vater zu lösen, von wenig Erfolg gekrönt waren - ausdrücklich erklärt er, dass die „Heiratsversuche“ aus dem Bemühen hervorgegangen seien, ihm auf diese Weise „zu entgehen“ (B 166) -, verstrickt er sich durch das Verfassen des Briefes noch tiefer in die Beziehung zu Hermann Kafka. Das Abfassen des Briefes, den er seinem Vater nie überreicht hat, vermag das Band der Abhängigkeit nicht zu lösen, das ihn mit seinem Vater verbindet. Obwohl er die befreiende Wirkung des Schreibens in seinem Brief eigens erwähnt - er erklärt hier: „Mein Schreiben handelte von Dir, ich klagte dort ja nur, was ich an Deiner Brust nicht klagen konnte.“ (B 160) -, spricht doch vieles dafür, dass ihm jener rauschhafte Zustand, den er etwa beim Schreiben seiner Erzählung Das Urteil erlebte, und der ihn die unheilvolle Verstrickung wenigstens vergessen ließ, weil er hier in eine eigene, abgeschlossene (Text-)Welt eintauchte $^{54}$, beim Verfassen des Briefes nicht vergönnt war. Denn nur beim Verfassen literarischer Texte nähert er sich wieder jenem exzessiven Schreiben an, das er in der Nacht vom 22. zum 23. September 1912 praktiziert hatte, und das ihm als Erlösung galt sowie als Maßstab für alles künftige Schreiben: „Diese Geschichte ,das Urteil' habe ich in der Nacht vom 22 zum 23 von 10 Uhr abends bis 6 Uhr früh geschrieben. Die vom Sitzen steif gewordenen Beine konnte ich kaum unter dem Schreibtisch hervorziehn. Die fürchterliche Anstrengung und Freude, wie sich die Geschichte vor mir entwickelte wie ich in einem Gewässer vorwärtskam. Mehrmals in dieser Nacht trug ich mein Gewicht auf

54 Vgl. hierzu Franz Kafka: „Das Urteil“, in: ders., Die Erzählungen und andere ausgewählte Prosa. Herausgegeben von Roger Hermes, Frankfurt/Main 2000, S. 47-60; Malcolm Pasley: „Der Schreibakt und das Geschriebene. Zur Frage der Entstehung von Kafkas Texten“, in: Claude David (Hg.), Franz Kafka. Themen und Probleme, Göttingen 1980, S. 9-25. 
dem Rücken. [...] Nur so kann geschrieben werden, nur in einem solchen $\mathrm{Zu-}$ sammenhang, mit solcher vollständigen Öffnung des Leibes und der Seele. “55

\section{Ergebnisse und Ausblick}

Bemüht man sich nun, den Ertrag einer Lektüre von Franz Kafkas Brief an den Vater zu skizzieren, die inspiriert ist von Pierre Bourdieus Theorie des sozialen Raumes, lassen sich fünf Themenfelder identifizieren, die eng miteinander verknüpft sind und mit den Stichworten ,List der pädagogischen Vernunft', ,Ausweitung des Erziehungsbegriffs', ,geschlechtsspezifische Sozialisation", ,Vaterbild" und ,familiale Reproduktion' bezeichnet werden können.

Kafkas Brief lässt sich in dieser Hinsicht als glänzende Fallstudie lesen, die an den unterschiedlichsten Beispielen das erläutert, was Bourdieu treffend die „List der pädagogischen Vernunft“ genannt hat. ${ }^{56}$ Deren besondere Kunst besteht darin, dass sie eine Verleiblichung der symbolischen Ordnung betreibt, die als solche meist unbemerkt bleibt, weil sie sich auf scheinbar nebensächliche und wenig bedeutsame Gegenstandsbereiche kapriziert. Im Medium vermeintlich harmloser Übungen, die sich in den Körper - hier: Franz Kafkas - einschreiben, wird dabei nicht nur die Übernahme der symbolischen Ordnung durch die Ausbildung einer entsprechenden inneren Haltung erzwungen, sondern auch die Ausprägung bestimmter Dispositionen angebahnt: Einer „Transsubstantiation“ gleich wird dergestalt „eine ganze Kosmologie, Ethik, Metaphysik und Politik vermittels so bedeutungsloser Befehle wie ,halt dich gerade“ oder ,halt das Messer nicht in der linken Hand “" übertragen. ${ }^{57}$

Die Ausweitung des Erziehungsbegriffes, die Jürgen Oelkers angemahnt hat, und die darauf abzielt, ihn nicht nur für jene intentional vollzogenen Handlungen zu reservieren, die sich eindeutig einem Erzieher zurechnen las$\operatorname{sen}^{58}$, lässt sich ebenfalls aus der Lektüre des Briefes als unverzichtbar ableiten: Franz Kafka beschreibt eindrucksvoll, dass die unheilvollen Wirkungen seines Vaters keineswegs nur von den gezielt vorgenommenen und absichtsvoll eingesetzten Erziehungsmaßnahmen im engeren Sinne ausgehen. Auch wenn er bei der Erörterung der „Erziehungsmittel“ (B 120) ${ }^{59}$ das nächtliche Aussetzen auf der Pawlatsche - einem Balkon - im frühen Kindesalter als fast schon traumatische Erfahrung beklagt, so sensibilisiert er doch dafür, dass es nicht selten unbeabsichtigte Handlungen, kaum reflektierte Verhaltensweisen

55 Zitiert nach R. Stach: Franz Kafka, S. 115.

56 Pierre Bourdieu: Entwurf einer Theorie der Praxis auf der ethnologischen Grundlage der kabylischen Gesellschaft, Frankfurt/Main 1976, S. 200.

57 Ebd.

58 Vgl. Jürgen Oelkers: Einführung in die Theorie der Erziehung, Weinheim, Basel 2001.

59 Vgl. Andreas Schmitz: „Zu Tode erzogen“ - Kindheit und Erziehung in Fritz Zorns Mars und Franz Kafkas Brief an den Vater. Unveröffentlichtes Manuskript, Bonn 2003. 
oder achtlose Umgangsformen seines Vaters waren, die ihn am stärksten trafen. Kafka selbst war sich dieser Wirkung offensichtlich bewusst: So nimmt er seinen Vater fast dessen besonderer Ausstrahlung gegenüber in den Schutz, als er in einer eigentümlich unpersönlichen Wendung von der „Erscheinung“ spricht, ,,in der Du auf das Kind wirktest“. (B 120)

Kaum weniger präzise sind Kafkas Beobachtungen jener sozialen Praktiken und Übungen, die für die geschlechtsspezifische Sozialisation verantwortlich sind: Es steht meines Wissens noch aus, den Brief an den Vater einer Lektüre zu unterziehen, die die unterschiedlichen Anstrengungen und Maßnahmen Hermann Kafkas auf die soziale Konstruktion von Männlichkeit hin interpretiert und systematisch auswertet. Nicht wenig spricht dafür, dass auf diese Weise ein weiterer, hochinteressanter Zugang zu Kafkas Auseinandersetzung mit seinem Vater gewonnen werden könnte. Gleichzeitig könnte auf diesem Wege auch der Gefahr begegnet werden, die Bedeutung der geschlechtsspezifischen Dimension des Habitus für Erziehungsprozesse, die Pierre Bourdieu in Die männliche Herrschaft in Form einer mikroskopisch genauen Interpretation der Eingangsszene von Virgina Woolfs To the Lighthouse herausgearbeitet hat ${ }^{60}$, noch länger zu vernachlässigen oder gar vollständig auszublenden.

Deutlich wird auf diese Weise auch, dass es durchaus problematisch ist, die Bedeutung des Vaters für die emotionale und kognitive Entwicklung eines Kindes zum Gegenstand einer wissenschaftlichen Reflexion zu machen, wenn dabei weder die historische Verfasstheit noch die soziale Rahmung des VaterKind-Verhältnisses systematisch in Rechnung gestellt wird. Die Strategie, entweder allein von der Erfahrung auszugehen, Einen Vater zu haben (Langeveld), und dies zum Ausgangspunkt anthropologisch angelegter, grundlegender Erörterungen über die Bedeutung des Vaters zu machen, oder diesen auf seine besondere Funktion für die psychische Entwicklung des Kindes hin zu untersuchen, wie dies von manchen psychoanalytischen Studien betrieben wird $^{61}$, steht daher - auch dies lässt sich aus Kafkas Text gewordener Selbsterforschung lernen - in der Gefahr, unterkomplexe und simplifizierende Reflexionsformen zu begünstigen.

Schließlich sensibilisiert Franz Kafkas Brief an den Vater auch dafür, dass die Durchquerung des sozialen Raumes in kürzester Zeit zu erheblichen innerfamilialen Verwerfungen führen kann: Die besondere Tragik, die auf dessen mehr als hundert eng beschriebenen Seiten zum Ausdruck kommt, besteht nicht zuletzt darin, dass Hermann Kafka den sozialen Aufstieg mit dem Ziel

60 Pierre Bourdieu: „Die männliche Herrschaft“, in: Irene Dölling/Beate Krais (Hg.), Ein alltägliches Spiel. Geschlechterkonstruktionen in der sozialen Praxis, Frankfurt/Main 1997, S. 153-217.

61 Vgl. hierzu etwa Martinus J. Langeveld: „Einen Vater zu haben“, in: Zeitschrift für Pädagogik 9 (1963), S. 1-19; Karl-Josef Pazzini: „Tertius datur. Skizze zur Funktion des Vaters in Bildung”, in: Werner Friedrichs/Olaf Sander (Hg.): Bildung/Transformation. Kulturelle und gesellschaftliche Umbrüche aus bildungstheoretischer Perspektive, Bielefeld 2002, S. 85-109. 
betreibt, die eigene Familie ökonomisch unabhängig zu machen und deren gefährdeten gesellschaftlichen Status abzusichern - und dass er damit unwissentlich die Krise der familialen Reproduktion heraufbeschwört, die bei Franz Kafka zur Ausprägung eines eigentümlich ,gespaltenen Habitus' führt und sich in einer kaum noch zu überbrückenden Entfremdung zwischen Vater und Sohn äußert. Es ist nicht auszuschließen, dass es diese Erfahrung ist, die in Franz Kafkas Notiz aus dem Januar 1917 anklingt:

„Ich war steif und kalt, ich war eine Brücke, über einem Abgrund lag ich, diesseits waren die Fußspitzen, jenseits die Hände eingebohrt, in bröckelndem Lehm hatte ich mich festgebissen. Die Schöße meines Rockes wehten zu meinen Seiten. In der Tiefe lärmte der eisige Forellenbach. Kein Tourist verirrte sich zu dieser unwegsamen Höhe, die Brücke war in den Karten noch nicht eingezeichnet. So lag ich und wartete; ich mußte warten; ohne abzustürzen kann keine einmal errichtete Brücke aufhören, Brücke zu sein.“62

\section{Literaturverzeichnis}

Barlösius, Eva: „„Das Elend der Welt‘. Bourdieus Modell für die „Pluralität der Perspektiven“ und seine Gegenwartsdiagnose über die ,neoliberale Invasion““, in: BIOS 12 (1999), S. 3-27.

Bateson, Gregory: Ökologie des Geistes. Anthropologische, psychologische, biologische und epistemologische Perspektiven, Frankfurt/Main 1992.

Bourdieu, Pierre: Entwurf einer Theorie der Praxis auf der ethnologischen Grundlage der kabylischen Gesellschaft, Frankfurt/Main 1976.

Bourdieu, Pierre: Was heißt Sprechen? Die Ökonomie des sprachlichen Tausches, hg. v. Georg Kremnitz, Wien 1990.

Bourdieu, Pierre: Die feinen Unterschiede. Kritik der gesellschaftlichen Urteilskraft, Frankfurt/Main 1992.

Bourdieu, Pierre: Sozialer Sinn. Kritik der theoretischen Vernunft, Frankfurt/Main 1993.

Bourdieu, Pierre: „Strukturalismus und soziologische Wissenschaftstheorie“, in: ders., Zur Soziologie der symbolischen Formen, Frankfurt/Main 1994, S. 7-41.

Bourdieu, Pierre: „Sozialer Raum und ,Klassen““, in: ders.: Sozialer Raum und ,Klassen'. Leçon sur la leçon. Zwei Vorlesungen, Frankfurt/Main 1995, S. 7-46.

Bourdieu, Pierre: „Ökonomisches Kapital - Kulturelles Kapital - Soziales Kapital“, in: ders., Die verborgenen Mechanismen der Macht. Schriften zu Politik \& Kultur 1, hg. v. Margareta Steinrücke, Hamburg 1997, S. 49-79.

62 Franz Kafka: Nachgelassene Schriften und Fragmente in der Fassung der Handschriften. Band I. Herausgegeben von Malcolm Pasley, Frankfurt/Main 1993, S. 304. 
Bourdieu, Pierre: „Die männliche Herrschaft“, in: Irene Dölling/Beate Krais (Hg.), Ein alltägliches Spiel. Geschlechterkonstruktionen in der sozialen Praxis, Frankfurt/Main 1997, S. 153-217.

Bourdieu, Pierre: „Widersprüche des Erbes“, in: ders. u. a., Das Elend der Welt. Zeugnisse und Diagnosen alltäglichen Leidens an der Gesellschaft, Konstanz 1997, S. 651-658.

Bourdieu, Pierre: „Familiensinn“, in: ders., Praktische Vernunft, Frankfurt/Main 1998, S. 126-136.

Bourdieu, Pierre: „Sozialer Raum, symbolischer Raum“, in: ders., Praktische Vernunft, Frankfurt/Main 1998, S. 13-32.

Bourdieu, Pierre u. a.: Das Elend der Welt. Zeugnisse und Diagnosen alltäglichen Leidens an der Gesellschaft, Konstanz 1997.

Cassirer, Ernst: Substanzbegriff und Funktionsbegriff. Untersuchungen über die Grundfragen der Erkenntniskritik, Berlin 1923.

Hierdeis, Helmwart (Hg.): „Lieber Franz! Mein lieber Sohn!“ Antworten auf Franz Kafkas „Brief an den Vater“, Wien 1997.

Kafka, Franz: Brief an den Vater. Faksimile. Herausgegeben und mit einem Nachwort versehen von Joachim Unseld, Frankfurt/Main 1994.

Kafka, Franz: „Das Urteil“, in: ders., Die Erzählungen und andere ausgewählte Prosa. Herausgegeben von Roger Hermes, Frankfurt/Main 2000, S. 47-60.

Kafka, Franz: Nachgelassene Schriften und Fragmente in der Fassung der Handschriften, Band I, hg. v. Malcolm Pasley, Frankfurt/Main 1993.

Koller, Hans-Christoph: Bildung und Widerstreit. Zur Struktur biographischer Bildungsprozesse in der (Post-) Moderne, München 1997.

Koller, Hans-Christoph: „Negativität und Bildung. Eine bildungstheoretisch inspirierte Lektüre von Franz Kafkas ,Brief an den Vater““, erscheint in: SG, Beiheft der Zeitschrift für Pädagogik, 2005.

Kremer, Deflef: Kafka. Die Erotik des Schreibens, Bodenheim 1998.

Langeveld, Martinus J.: „Einen Vater zu haben“, in: Zeitschrift für Pädagogik 9 (1963), S. 1-19.

Mollenhauer, Klaus: Vergessene Zusammenhänge. Über Kultur und Erziehung, Weinheim, München 1998.

Oelkers, Jürgen: „Kindheit als Glück und als Geißel: Fontanes und Kafkas Erinnerungen im Vergleich“, in: ders., Die Herausforderung der Wirklichkeit durch das Subjekt. Literarische Reflexionen in pädagogischer Absicht, Weinheim, München 1985, S. 21-53.

Oelkers, Jürgen: „Vater? Vater!“, in: Helmwart Hierdeis (Hg.), „Lieber Franz! Mein lieber Sohn!“ Antworten auf Franz Kafkas „Brief an den Vater“, Wien 1997, S. 205 216.

Oelkers, Jürgen: „Väter und Söhne: Über Anklage, Mißverständnis und den fehlenden Adressaten in der Erziehung“, in: Neue Sammlung 38 (1998), S. 533-553.

Oelkers, Jürgen: Einführung in die Theorie der Erziehung, Weinheim, Basel 2001. 
Pasley, Malcolm: „Der Schreibakt und das Geschriebene. Zur Frage der Entstehung von Kafkas Texten“, in: Claude David (Hg.), Franz Kafka. Themen und Probleme, Göttingen 1980, S. 9-25.

Pawel, Ernst: Das Leben Franz Kafkas, München, Wien 1986.

Pazzini, Karl-Josef: „Tertius datur. Skizze zur Funktion des Vaters in Bildung“, in: Werner Friedrichs/Olaf Sander (Hg.), Bildung/Transformation. Kulturelle und gesellschaftliche Umbrüche aus bildungstheoretischer Perspektive, Bielefeld 2002, S. 85109.

Rieger-Ladich, Markus: Mündigkeit als Pathosformel. Beobachtungen zur pädagogischen Semantik, Konstanz 2002.

Rieger-Ladich, Markus: „Unbequeme Zaungäste? Pierre Bourdieu und Niklas Luhmann beobachten die Familie und irritieren die Erziehungswissenschaft", in: Vierteljahrsschrift für wissenschaftliche Pädagogik 80 (2004), S. 85-102.

Rieger-Ladich, Markus: „Böse Blicke. Streifzüge durch das Feld der Erziehungswissenschaft mit Pierre Bourdieu“, in: Jan Masschelein/Wolfgang Nieke/Ludwig Pongratz (Hg.), Kritik der Pädagogik - Pädagogik als Kritik, Opladen 2004, S. 134-155.

Robert, Marthe: Einsam wie Franz Kafka. Aus dem Französischen von Eva MichelMoldenhauer, Frankfurt/Main 1985.

Schärf, Christian: Franz Kafka. Poetischer Text und heilige Schrift, Göttingen 2000.

Schmitz, Andreas: „Zu Tode erzogen“ - Kindheit und Erziehung in Fritz Zorns Mars und Franz Kafkas Brief an den Vater, unveröffentlichtes Manuskript, Bonn 2003.

Stach, Reiner: Franz Kafka. Die Jahre der Entscheidungen, Frankfurt/Main 2002.

Stierlin, Helm: Individuation und Familie. Studien zur Theorie und therapeutischen Praxis, Frankfurt/Main 1989.

Wagnerová, Alena: „Im Hauptquartier des Lärms“. Die Familie Kafka aus Prag, Köln 1997. 